\title{
Research of Transport Junctions of Flow Analysis Algorithm based on Decision- making Theory
}

\author{
Xiao-feng Li \\ Department of Computer Science and \\ Technology \\ ChengDong College Of Northeast \\ Agricultural \\ Harbin ,150001, china \\ E-mail:mberse@126.com
}

\author{
Shu-chun Ding \\ Department of Communication \\ Engineering \\ Electronic Engineering college of \\ Heilongjiang university, \\ Harbin , 150001,china \\ E-mail:dingshuchun@126.com
}

\author{
Wang Bin*( Author for \\ correspondence) \\ College of Information Science \& \\ Electronic Technology \\ Jiamusi university, Jiamusi, \\ Jiamusi,154007,China; \\ E-mail:jmsuwang@163.com
}

\begin{abstract}
Intersection traffic flow analysis and analysis of algorithms, design of algorithms, this paper, the vehicles unified identification as a standard car equivalent steps of the algorithm based on the decision-making on the flow of traffic junctions, the algorithm is applicable not only to single-coil detector or single magnetic detector such as a single detector, but also applies to dual-loop detectors. The detection process is simple and can achieve very high accuracy rate, and through case studies, to verify the effectiveness and accuracy of change algorithm
\end{abstract}

Keywords-component; Traffic intersection; standard dolly equivalent; coil; flow analysis; decision-making

\section{INTRODUCTION}

With the development of social economy and improvement of people's living standards, an increasing demand for transportation is witnessed and thus issues on urban transportation arouse more and more attentions from the public. Of all those problems, the most significant refers to how to realize the organized unification of people, cars and roads, as to effectively prevent traffic congestion, reduce road accidents and abate pollution[1]. Traffic flow at crossroads is generally represented by passenger car equivalent[2]. So far, the widely used approach to measure traffic-flow rate is single loop detection, while double loop detection is typically used for higher precision [3].

With regards to transport junctions of flow analysis algorithm, there are problems in these stages of data acquisition, pre-processing and feature extraction. In the stage of collecting data, major factors affecting the frequency variation of oscillator circuit of loop detector are chassis and speed. When the chassis becomes uneven and speed is ever changing, it would interfere with frequency waveform [4]. Secondly, because of detector, environment, used method and human element, the original measurement data inevitably have errors despite some software algorithms can process them. Yet, existing algorithms still have difficulties on system errors and gross errors. Then in the period of data pre-processing, asymmetrical base plate would cause frequency waveform in unnoticeable boundary, demanding multiple processing, such as quadratic differentials, reduction of dimensionality. It is certain to make wrong judgment about vehicle type [5].
Based on so many problems and shortcomings mentioned above, it discussed the transport junctions of flow analysis algorithm and introduced a method based on decisionmaking theory. The proposed solution is not only applicable for single detector like single loop detector or single magnetic detector, and also suitable for dual loop detector. It is simple and can help achieve an extremely high accuracy rate, with very good cost-performance ratio.

\section{BASIC CONCEPTS}

\section{A. Single loop detection}

When a car approaches coils, inductance value will vary. Upon finding such variation, the vehicle detector will output a signal whose logic is true to indicate car's accession to coils, which is nominally vehicle occupancy [6]; once inductance value restores to the one for free occupancy when the car leaves coils, the detector exports instantly a signal whose logic is false, referring to car's departure from them. Then, import a counter signals sent from vehicle detector and the result concluded by it is traffic rate flowing through the loop. Obviously, it is impossible to determine the type of vehicle through this method and further the passenger car equivalent can't be obtained either, much less accurate detection of traffic volume of transport junctions.

\section{B. Dual loop detection}

This detection method is utilized where two circular coils of the same properties are buried along the direction of traffic flow and the centre distance of those coils is three to five meters [7]. When cars run through both coils, the inductance value will change. Cars' passing status is detected and results are transmitted to vehicle detector for acquisition and calculation. This method computes speed with the use of intervals by vehicle passing both coils. With the speed and such time difference, vehicle length is acquired. Based on that it is easy to classify them and finally traffic flow is expressed in the form of the passenger car equivalent. 


\section{DESIGN OF TRANSPORT JUNCTIONS OF FLOW ANALYSIS ALGORITHM BASED ON DECISION-MAKING THEORY}

\section{A. Design philosophy}

In the event big errors are observed in the single loop detection method, the installation of dual loop coils is costly and it's difficult to install and manage those coils, we present a new approach-transport junctions of flow analysis algorithm based on decision-making theory, for the purpose of measuring accurately the throughput at intersections and reacting promptly to the obstruction before further measures are taken.

\section{B. Steps}

The proposed method includes the following steps:

Step one: the control unit detects whether there are cars passing by from the detector through real-time scanning with the advantage of single loop detector placed before the stop line of lane crossing. The unit records level state "1" for cars, otherwise " 0 "; at the same time, the control unit stores state levels at each scanning time in the memory device.

Step two: when detection begins, the control unit resets traffic flow $\mathrm{N}$ into " 0 " and synchronously checks the level state of the current scanning moment. If it's " 0 ", calculate passing time $\triangle \mathrm{t}$ of vehicle as per (i); if it's "1", calculate $\triangle \mathrm{t}$ as per (ii).

(i) The control unit sequentially looks up the level state of subsequent scanning time. if it is " 1 ", mark its scanning time is marked "ta"; then, the unit continues checking. If the level state is " 0 ", write down its scanning time as "tb". In the meantime, the control unit reckons passing time of vehicle, i.e. $\triangle \mathrm{t}=\mathrm{tb}$-ta.

(ii) The control unit examines via the storing device the state level before the current scanning time in reverse order. If it is " 0 ", the scanning time is correspondingly recorded "ta"; next, the control unit sequentially checks the state level after the current scanning moment. If it is " 0 ", the time is "tb". And simultaneously the control unit computes passing time of vehicle, as $\triangle \mathrm{t}=\mathrm{tb}$-ta- 0.25 .

Step three: according to the type of a lane and the lasting time of green lights of the lane, the control unit converts passing time $\triangle \mathrm{t}$ into traffic flow increment $\triangle \mathrm{N}$. If it is left-turn lane, the conversion is made as per (iii); and (iv) and (v) for respectively right-turn and through lanes.

(iii) If green lights of the lane are on and $0.25 \mathrm{~s} \leq \triangle \mathrm{t} \leq 3.75 \mathrm{~s}$, then $\triangle \mathrm{N}=1 ; 4 \mathrm{~s} \leq \triangle \mathrm{t} \leq 7.5 \mathrm{~s}$, then $\triangle \mathrm{N}=2 ; 7.75 \mathrm{~s} \leq \triangle \mathrm{t}$, then $\triangle \mathrm{N}=3$. When red or yellow lights are on, $\triangle \mathrm{N}=0$; (iv) If the lasting time of green lights of the lane is less than or equal to $10 \mathrm{~s}$, and $0.25 \mathrm{~s} \leq \Delta \mathrm{t} \leq 4 \mathrm{~s}$, then $\triangle \mathrm{N}=1 ; 4.25 \mathrm{~s} \leq \triangle \mathrm{t} \leq 10 \mathrm{~s}$, then $\triangle \mathrm{N}=2$; if that time is $>10 \mathrm{~s}$, and $0.25 \mathrm{~s} \leq \triangle \mathrm{t} \leq 3.5 \mathrm{~s}$, then $\triangle \mathrm{N}=1$; $3.75 \mathrm{~s} \leq \triangle \mathrm{t} \leq 6.25 \mathrm{~s}$, then $\triangle \mathrm{N}=2 ; 6.5 \mathrm{~s} \leq \triangle \mathrm{t} \leq 10 \mathrm{~s}$, then $\triangle \mathrm{N}=3$; if red or yellow lights of the lane are on, then $\triangle \mathrm{N}=0$;

If the lasting time of green lights of the lane is less than or equal to $5 \mathrm{~s}$, and $0.25 \mathrm{~s} \leq \triangle \mathrm{t} \leq 2.5 \mathrm{~s}$, then $\triangle \mathrm{N}=1 ; 2.75 \mathrm{~s} \leq \Delta \mathrm{t} \leq 5 \mathrm{~s}$, then $\triangle \mathrm{N}=2$; if that time is $>5 \mathrm{~s}$, and $0.25 \mathrm{~s} \leq \triangle \mathrm{t} \leq 2 \mathrm{~s}$, then $\triangle \mathrm{N}=1$; $2.25 \mathrm{~s} \leq \triangle \mathrm{t} \leq 2.5 \mathrm{~s}$, then $\triangle \mathrm{N}=2 ; \quad 2.75 \mathrm{~s} \leq \triangle \mathrm{t} \leq 3.75 \mathrm{~s}$, then $\triangle \mathrm{N}=3 ; 4 \mathrm{~s} \leq \triangle \mathrm{t} \leq 5 \mathrm{~s}$, then $\triangle \mathrm{N}=4 ; 5.25 \mathrm{~s} \leq \triangle \mathrm{t}$, then $\triangle \mathrm{N}=5$; if red or yellow lights of the lane are on, then $\triangle \mathrm{N}=0$ 。

Step four: the control unit adds the present value of traffic volume $\mathrm{N}$ and $\triangle \mathrm{N}$ obtained in step three; then gives it to $\mathrm{N}$.

Step five: calculate the passing time of following vehicle as per (i) in Step two; then, convert it as per Step three to get the increment $\triangle \mathrm{N}$, at last, update $\mathrm{N}$ by repeating Step four.

Step six: repeat Step five till detection ends. When it is over, the resulted $\mathrm{N}$ is traffic flow for the detection time.

\section{EXPERIMENT AND RESULTS DISCUSSION}

\section{A. Preparations}

Before the use of the algorithm, it is necessary to do something about the placement of detectors.

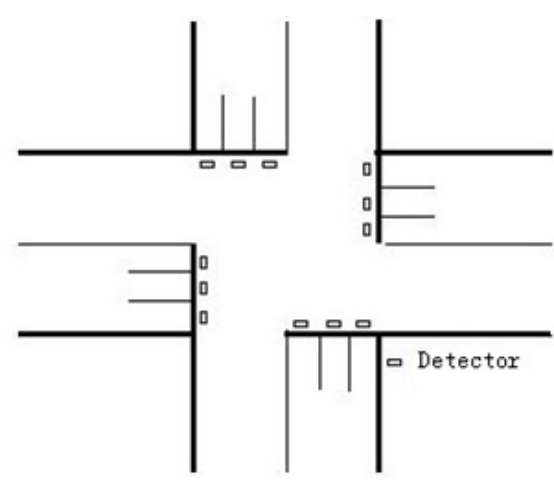

Figure 1. Detector placement

The control unit scans in real-time whether there are cars through single detector placed before the stop line of driveway crossing (Fig. 1). The level state "1" means cars 
and " 0 " means no cars. At the same time, the unit inputs the level state of each scanning moment into memory device.

In practical terms, the detector can be single loop or single magnetic. The scanning interval is at most $0.25 \mathrm{~s}$. The control unit can be single chip microcomputer (SCM), industrial personal computer (LPC), or programmable logic controller (PLC).

Since detected and undetected signals by the detector are apparently different from each other [8], the control unit can accurately determine whether there are cars passing by from the detector, ensuring no any misjudgment.

\section{B. Experimental analysis}

The experiment is carried out in the intersection of Minjiang Road (East-West passageway) and Wudingqiao Blvd. (South-North passageway) in Beijing. Each road consists of one protective left-turn lane, two through-traffic lanes and one right-turn lane. The width is 3.5 meters.

Types of vehicle: cars, a few buses and minivans

Experimental process: choose sunny, rainy or foggy days to carry out the following process for several times in preset time: (1) use the example method to detect traffic flow represented by passenger car equivalent, $\triangle N=0$ for mini-passenger car, $\triangle N=1$ for minivans and $\triangle N=2$ for buses. Count the value of traffic flow every ten seconds; (2) enumerate manually the number of the actual passing vehicle every ten seconds with roadway surveillance cameras and then convert it to traffic flow in the form of passenger car equivalent. Results are shown in Fig. 2 and 3.

It reveals that the proposed method proves to reach the accuracy rate of at least $95 \%$.

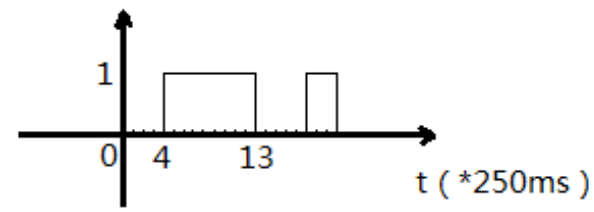

Figure 2. Detector scanning ordinary diagram

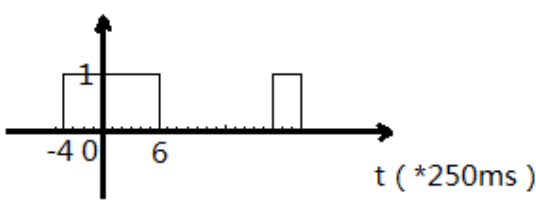

Figure 3. Detector scan showing the results of the use of the algorithm
Besides, the algorithm is applied to implement the above process in 1500 s during a rush-hour period. The number of measured cars is 141 , while manual statistics show 148 cars, therefore, the accuracy rate is $95.27 \%$.

\section{Results}

The algorithm is demonstrated to be superior to existing methods after comparison:

(i) The detection process is simple and easy. It has a rather high rate of precision and is cost-effective; since the detector is buried before the stop line, cars won't stop over it and they can pass by the stop line at steady speed, no big variations of frequency and range, which is helpful to acquire accurate data;

(iii) acquisition data and generated data are of low dimension. Small data size won't cause too many troubles;

(iv) it's possible to get directly the traffic flow in passenger car equivalent, no need of vehicle type, which avoids problems arising out of unrecognized types and thus the accuracy will keep at a high rate but not be influenced by base plate, detection environment and so on;

(v) it has wide scope of applications. Single detector is applicable, such as single loop detector or single magnetic detector.

\section{CONCLUSIONS}

The innovative point of this paper is that it presents transport junctions of flow analysis algorithm based on the decision-making theory. The method overcomes a lot of difficulties in existing ones. The single loop detection method can effectively improve the precision of measured traffic flow rate. Experiments performed on roads of Beijing justifies that the method is meaningfully referential to traffic jams in the city.

\section{REFERENCES}

[1]Tanguo Xian,Yaoshu Shenand Huang Zhen.Short term traffic flow modeling and forecasting of urban intersection.Computer and Communications, 2005:12-18

[2]Daihong.Traffic information processing technology based on fuzzy theory. Jilin University,2007

[3]Liu Yingying, LiuChuan.Intersection-related traffic prediction algorithm. Journal of Qingdao University of Science and Technology ,2004:23-28

[4] Li jianwu chen-fa. Short-term traffic flow forecast of the road network based on rough set theory and support vector machine. Application Research of Computers,2010:67-70 
[5] Zhang Yumei,Qu Shiru.Temperature chaotic characteristics of the traffic flow analysis and prediction model.Civil Engineering Journal,2009:45-49

[6]ZhangHe, WangWei, GuHuaizhong. Application of cluster analysis and stepwiseregression in predicting the traffic volume of lanes.Journal of Southeast University, 2005, 21(3):359-362.

[7]SohallNadimBirBhanu.Physiealmodelsformovingshadowandobjeetdetee tioninvideo.IEEE.TransactionsonpatternAnalysisandMaehineIntelligenee, 2 008,26(8):1079-1057
[8] Stauffer C, Grimson W. Learning pattern of aetivity using realtimetraeking.IEEE Transaction Son Patter Analysis and Maehine Intelligenee,2003,22(8):747-757 